\title{
Las destrezas argumentativas en la evolución de modelos en una actividad de geología
}

\author{
Paloma Blanco Anaya \\ Departamento de Didácticas Aplicadas, Universidade de Santiago de Compostela. España. \\ paloma.blanco@usc.es \\ ORCID: https:// orcid.org/0000-0002-3347-1103 \\ Joaquín Díaz de Bustamante \\ Departamento de Didácticas Aplicadas, Universidade de Santiago de Compostela. España. \\ joaquin.diaz.1952@gmail.com \\ ORCID: https:/ / orcid.org/0000-0001-6042-2211
}

\section{Paula Cristina Cardoso Mendonça}
Departamento de Quimica e Programa de Pós-graduação em Educação da Universidade Federal de Ouro
Preto.Brasil.paulamendonca@ufop.edu.br
ORCID: bttps:/ / orcid.org/0000-0003-1762-4840

[Recibido: 18 Diciembre 2018. Revisado: 13 Marzo 2019. Aceptado: 7 Junio 2019]

\begin{abstract}
Resumen: Este artículo presenta un análisis sobre cómo las destrezas argumentativas intervienen en el proceso de modelización. Para ello se hace una revisión de la literatura sobre los trabajos en los que se analizan las interacciones entre ambas prácticas científicas, lo cual establece un marco para fundamentar el objetivo de investigación: examinar cómo evolucionan los modelos de los estudiantes en el proceso de elaboración y evaluación de los mismos a través de las destrezas argumentativas. El estudio se llevó a cabo con alumnado de $1^{\circ}$ de bachillerato que cursaba Biología y Geología (16-17 años), quienes tenían que explicar de forma científica cómo se formó el pliegue sinclinal de O Courel (Lugo, España). Los resultados muestran que las destrezas argumentativas con mayor implicación en la evolución de los modelos son la contraargumentación y, sobre todo, la refutación. Para favorecerlo es necesario que en el diseño de actividades de modelización se incluyan aspectos que favorezcan el desempeño conjunto de las dos prácticas científicas, aportando datos para evaluar los modelos y favorecer la crítica y el intento de persuasión entre compañeros, lo cual enriquecerá las destrezas argumentativas.
\end{abstract}

Palabras clave: Modelización; modelos; argumentación; prácticas científicas; geología.

The argumentative skills in the evolution of models in a geology activity

Abstract: This article presents an analysis about how argumentative skills take part in the modelling process. To do this, a literature review about previous studies that analyse the interactions between both scientific practices was done, which establishes a framework to support the research objective: to examine how students' models evolve in the process of elaboration and evaluation of them through the argumentative skills. The study was carried out with students from the nine grade of high school studying Biology and Geology (16-17 years old), who had to explain scientifically how the O Courel syncline (Lugo, Spain) was formed. The results show that the argumentative skills with greater implication for the evolution of the models are counter-argumentation and, above all, refutation. To achieve this it is necessary that the design of modelling activities includes aspects that favor the performance of the two scientific practices together, such as providing data to evaluate the models and favor the criticism attempt to persuade among classmates, which enrich the argumentative skills.

Keywords: Modelling, models, argumentation, scientific practices, geology

Para citar este artículo: Blanco Anaya, P., Díaz de Bustamante, J. y Mendonça, P. C. C. (2019) Las destrezas argumentativas en la evolución de modelos en una actividad de geología. Revista Eureka sobre Enseñanza y Divulgación de las Ciencias 16(3), 3105. doi: 10.25267/Rev_Eureka_ensen_divulg_cienc.2019.v16.i3.3105 


\section{Introducción}

En la literatura de didáctica de las ciencias parece haber consenso en que la enseñanza y aprendizaje de las ciencias debe girar en torno a que el alumnado sea capaz de desarrollar las prácticas científicas (Osborne 2014; Jiménez-Aleixandre y Crujeiras 2017). Las prácticas científicas tienen una naturaleza social, son practicadas en las comunidades científicas e incluyen constructos, identidades, valores, lenguas y herramientas intelectuales y discursivas específicas de los campos disciplinares (Stroupe 2015). Entendemos que las prácticas científicas escolares guardan relaciones de semejanza con aquellas practicadas por los científicos. Según Osborne (2011), las principales prácticas científicas introducidas en el ámbito escolar comprenden: investigar (indagación), desarrollar explicaciones y soluciones (modelización) y evaluar (argumentación). Trabajar con prácticas científicas se muestra relevante porque favorece la comprensión de los estudiantes acerca de cómo sabemos lo que sabemos, en términos de las pruebas que fundamentan la elección de modelos alternativos. Además, favorece el entendimiento epistémico y la vivencia social de los procesos involucrados en la elaboración y evaluación del conocimiento (Jiménez Aleixandre 2010; Osborne 2014). De forma general, existe una relación intrínseca entre estas prácticas científicas, dado que la indagación contribuye a la elaboración y revisión de modelos y, a su vez, el uso de pruebas en el discurso argumentativo ayuda a la elaboración, revisión y evaluación del modelo (Jiménez Aleixandre, Bravo y Puig 2009).

En este estudio nos centramos en detallar la relación entre las prácticas de modelización y argumentación, pues entendemos que son dependientes una de la otra en el sentido de que 1) la modelización y el uso de modelos respaldan teóricamente las justificaciones de los argumentos y 2) el uso de datos y pruebas, durante el proceso de argumentación, permite evaluar la adecuación del modelo empleado para explicar un fenómeno científico.

Dada la reciente literatura que recoge estudios en los que se analizan las prácticas científicas de argumentación y modelización conjuntamente (Mendonça y Justi 2013; Puig, Ageitos y Jiménez-Aleixandre 2017), o bien se detallan actividades en que se afirma que ambas prácticas están integradas (Evagorou y Puig 2017; Ageitos, Puig y Calvo 2017), se considera relevante examinar cómo estas prácticas co-ocurren para conocer cómo la modelización y argumentación se complementan en el contexto de la investigación escolar. Es por ello que, este estudio contribuye al conocimiento sobre cómo estas prácticas científicas se articulan durante el discurso oral de los estudiantes mientras resuelven un problema.

El objetivo de este trabajo consiste en examinar, en el contexto de la resolución de un problema de geología, cómo evolucionan los modelos de los estudiantes en el proceso de elaboración y evaluación de los mismos a través de las destrezas argumentativas.

\section{Argumentación y modelización: punto de encuentro}

\section{Aspectos previos sobre la argumentación}

La argumentación es considerada como el proceso discursivo que permite la construcción de conocimiento, ya sea individual o colectivamente, y su evaluación mediante las pruebas disponibles, tanto teóricas como empíricas (Erduran y Jiménez-Aleixandre 2008). Esta práctica incluye destrezas argumentativas (Kuhn 1991; Justi 2015) tales como: usar pruebas, elaborar argumentos, contraargumentar, elaborar teorías alternativas y refutar, las cuales se comentan a continuación.

El término prueba hace referencia a cualquier dato (conjunto de información de la que se dispone, la cual puede estar en diferentes formatos) empleado para mostrar que un enunciado es cierto o falso (Jiménez Aleixandre 2010). Ese dato debe ser interpretado para que adquiera 
la función de prueba, pues coincidiendo con Koslowski, Marasia, Chelenza y Dublin (2008), un dato es considerado como una prueba cuando es incorporado en una justificación. Sin embargo, la integración del dato para ser empleado como prueba depende directamente del paradigma teórico de la persona que lo interpreta, lo cual se integra en el conocimiento básico que respalda a la justificación dentro del argumento (Toulmin 1985). Es por ello que aquí se intuye una de las relaciones entre la argumentación y la modelización, pues el modelo teórico subyace en la justificación del argumento.

En la elaboración de argumentos lo relevante es la búsqueda de una justificación que fortalezca la conclusión. Como las pruebas pueden ser interpretadas de diferentes formas, dando lugar a conclusiones diferentes, los sujetos elaboran contraargumentos o teorías alternativas (Kuhn 1991). Según la autora, la refutación consiste en las críticas a los datos o evidencias que sostienen el argumento del oponente (así como las teorías alternativas o contraargumentos de los oponentes).

Estas destrezas argumentativas contribuyen a la evaluación del conocimiento (Erduran y Jiménez-Aleixandre 2008) que se elabora durante el proceso de modelización porque incluye tanto los procesos de elaboración, como de críticas a las conclusiones o a las explicaciones científicas (Justi 2015).

\section{Aspectos previos sobre la modelización}

En el ámbito de la didáctica de las ciencias, el término modelo se emplea para hacer referencia a las representaciones con las que explicamos y visualizamos los fenómenos científicos (Gilbert, Boulter y Elmer 2000; Galagovsky y Adúriz-Bravo 2001; Schwarz et al. 2009; entre otros). Lo que permite esa representación es el hecho de que los modelos están conformados por elementos que pueden ser identificados con aspectos del mundo real (Giere 2004). En sí, los modelos engloban un conjunto de ideas o teorías de manera que una teoría puede ser representada por un modelo, o bien, varios modelos pueden conformar una teoría (Gilbert y Justi 2016).

Dentro de la clasificación de modelos establecida por Gilbert, Pietrocola, Zylbersztajn y Franco (2000), destacamos para este estudio el modelo mental y el expresado, dado que el análisis del modelo de los estudiantes debe realizarse mediante el modelo expresado, puesto que su modelo mental es inaccesible para nosotros. El modelo expresado se puede manifestar mediante diversos modos de representación: concreto o material (modelos materiales, maquetas), verbal (descripción, explicación, analogía y metáfora) y visual (diagramas, animaciones, simulaciones) considerando la clasificación de Boulter y Buckley (2000). Esta representación del modelo nos permite tener una aproximación a cómo el alumnado se apropia del conocimiento científico a través de lo que expresa oralmente, escribe o dibuja (Márquez y Artés, 2016).

Además de permitimos explicar los fenómenos científicos, en general, los modelos se caracterizan por: 1) facilitar la visualización de procesos y entidades abstractas (Oliveira, Justi y Mendonça 2015), 2) formular predicciones sobre los fenómenos (Franco y Colinvaux 2000; Schwarz et al. 2009), 3) generar nuevas ideas (Gilbert y Justi 2016) y 4) están limitados por las visiones del mundo y creencias de las personas (Franco y Colinvaux 2000).

Considerando la enseñanza basada en las prácticas científicas, es importante que los estudiantes evalúen sus propios modelos modificándolos o rechazándolos en lo que se conoce como modelización (Justi y Gilbert 2002; Oliva 2011). El proceso de modelización propuesto por Justi y Gilbert (2002), en el diagrama del modelo de modelización (MMD), consta de cuatro etapas que comprenden: elaboración (mental), expresión (según diferentes modos de representación), comprobación (mental o empírica) y evaluación del modelo. Dado que en el 
proceso de modelización la relación entre las etapas no sigue un orden determinado, los autores propusieron una versión del modelo de modelización en el que las cuatro fases están conectadas entre sí (Gilbert y Justi 2016).

\section{Punto de encuentro: la articulación de la modelización y de la argumentación}

Desde la perspectiva de la argumentación ésta presenta un papel relevante en la elaboración de explicaciones y modelos (Siegel 1989), del mismo modo que ocurre cuando los estudiantes tienen que conectar los datos con las conclusiones mediante una justificación, respaldada por el conocimiento básico (Toulmin 1958) en el cual subyace un modelo teórico. O dicho de otro modo, los estudiantes serán capaces de unir los datos con las conclusiones, es decir, de transformarlos en prueba, si poseen los conocimientos teóricos relacionados con los contenidos que se estén abordando en el problema propuesto.

Esta inclusión de los modelos en la estructura del argumento ha sido propuesta por McNeill y Krajcik (2012), quienes han integrado la construcción de explicaciones, la argumentación y el modelo de argumentación de Toulmin (1985) para generar lo que denominan explicación científica. Este término engloba la conclusión, las pruebas y las refutaciones, sin embargo, añaden otro elemento: el razonamiento. Según estos autores el razonamiento permite la unión de las pruebas con la conclusión, al explicar por qué la prueba apoya a la conclusión. Es decir, el "razonamiento es una justificación que conecta las pruebas con la conclusión" (ZembalSaul, McNeill y Hershberger 2013, p.24).

Lo importante del razonamiento es que debe estar apoyado en modelos científicos (Clement 1989), lo cual concuerda con la rúbrica elaborada por McNeill y Krajcik (2008) en la que se evalúan las explicaciones científicas según los conocimiento científicos que integra. Para entender la articulación entre la modelización y la argumentación, consideramos relevante la progresión que establecen en los razonamientos, pues la evolución de una explicación se debe al empleo de conocimientos científicos o, en nuestra opinión, modelos con los que se apoya una conclusión.

Mediante el uso de pruebas se puede determinar si el modelo se ajusta a los datos de los que se dispone (Böttcher y Meisert 2011; Justi 2015). Además, en el caso de que los estudiantes tengan que elaborar una explicación para un fenómeno en concreto, deberán apoyarse en los modelos teóricos que poseen, así como de ser capaces de determinar qué explicación es la más adecuada según el contexto y la información disponible (Reiser, Berland y Kenyon 2012; Osborne 2011).

Passmore y Svoboda (2012) consideran que los modelos presentan un papel central en la elaboración de explicaciones mediante el uso de patrones de datos (estructuración de los datos relevantes para el fenómeno en cuestión). Como señalan, en este proceso existen al menos cuatro aspectos en el proceso de modelización en los que la argumentación tiene lugar: 1) al determinar qué investigar, 2) al elaborar el diseño de la investigación, 3) al emplear un modelo para explicar un fenómeno y 4) al juzgar los modelos e ideas que se están comparando.

De lo comentado previamente se puede resumir que la relación entre la modelización y argumentación se hace evidente cuando:

1) los modelos empleados se adecúan al conocimiento científico y a las pruebas disponibles (Böttcher y Meisert 2011; Gilbert y Justi 2016),

2) el proceso de argumentación ayuda a evaluar si el modelo empleado en una explicación es acorde con esas pruebas, lo cual requiere de destrezas como refutar, contraargumentar, o bien persuadir para llegar a consensuar qué modelo permite una mejor explicación del fenómeno (Oliveira, Justi y Mendonça 2015); 
3) para establecer un patrón de datos con la información disponible tiene que haber un modelo que subyace (Passmore y Svoboda 2012).

Por ello, desde nuestro punto de vista, el vínculo entre la modelización y la argumentación se hace evidente en el marco de las explicaciones científicas. Los nexos entre estas prácticas se pueden resumir gráficamente en la figura 1, en la cual se muestra que el aporte de la modelización a la argumentación se debe a que los modelos sirven para guiar y apoyar las justificaciones de los argumentos, así como que la argumentación contribuye a evaluar la adecuación de los modelos al contexto o a los datos disponibles. Finalmente, ambas prácticas confluyen generando una explicación final, elaborada al amparo de los modelos teóricos y evaluada a la luz de las pruebas.

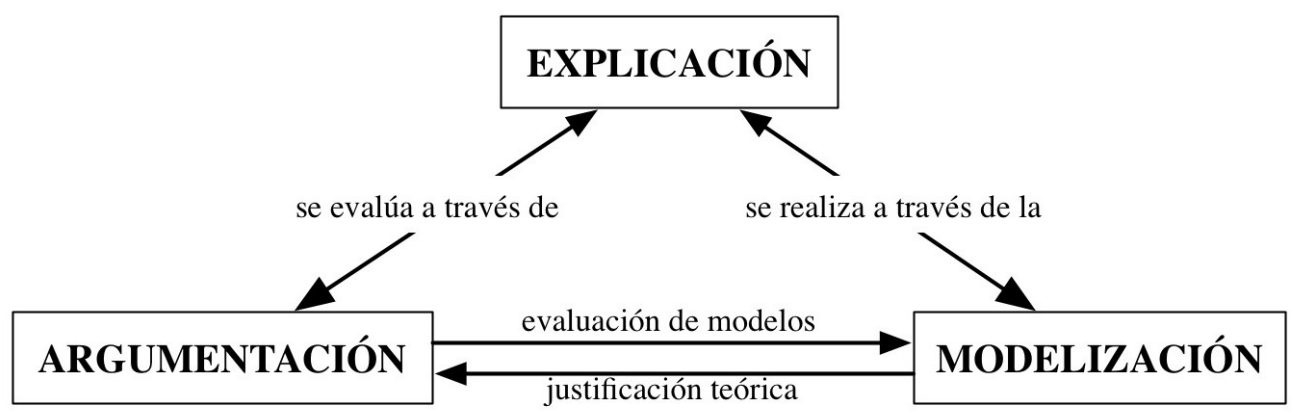

Figura 1. Relación entre las prácticas de modelización y argumentación en el proceso de generación de una explicación científica (elaboración propia).

Aunque los estudios previos pongan de manifiesto la relación entre la argumentación y la modelización, actualmente se han encontrado escasos trabajos que se centren en el análisis de ambas prácticas simultáneamente. A continuación se resumen estos trabajos destacando sus aportaciones a la relación de la argumentación y modelización.

Böttcher y Meisert (2011) han desarrollado una herramienta de análisis para conocer cómo se desarrolla la argumentación en las fases de la construcción del modelo, tomando para ello la propuesta de Clement (2008). Estos autores analizan la presencia de la argumentación durante el proceso de evolución desde el modelo inicial hasta el modelo objetivo que, según Clement (2008) y siguiendo la terminología de Gilbert, Boulter y Elmer (2000), constituiría el modelo curricular, o lo que es lo mismo, el modelo que se espera que los estudiantes dominen después de la instrucción.

Böttcher y Meisert (2011) indican que la argumentación consiste en el uso de datos empíricos para evaluar críticamente la similitud de los modelos con el mundo real que se pretende representar por medio de ellos.

Mendonça y Justi (2013) analizaron cómo los estudiantes llevaban a cabo ambas prácticas mientras modelaban los enlaces iónicos y las interacciones moleculares, llegando a la conclusión de que la argumentación había tenido lugar en todas las fases de la modelización (tomando como base el MMD de Justi y Gilbert 2002), desde la producción de un modelo inicial hasta su evaluación, así como en la consideración de las limitaciones del modelo. Esto aporta una nueva perspectiva, pues estudios previos como el de Clement (2008) y Böttcher y Meisert (2011) indicaban que la argumentación era importante sobre todo en la fase de evaluación del modelo. En un artículo posterior, Mendonça y Justi (2014) analizaron qué tipos de argumentos eran propuestos por los estudiantes durante la modelización, así establecieron tres niveles para clasificar los argumentos: 1) justificaciones aisladas, 2) conexiones complejas entre la justificación y la prueba, y 3) conexiones complejas entre la justificación y la prueba pero con una finalidad persuasiva. De este último estudio han obtenido que los argumentos 
del nivel 1 se encontraron en las fases iniciales del proceso de modelización, producción y expresión, en función de que los alumnos se encontraban en un proceso de elaboración de los significados sobre los temas químicos abordados; mientras que los niveles los niveles 2 y 3 han sido más recurrentes durante la comprobación y la evaluación de los modelos cuando los alumnos buscaron un consenso sobre los significados analizando las distintas explicaciones.

Puig, Ageitos y Jiménez-Aleixandre (2017) centraron la investigación en el análisis acerca de cómo la modelización y la argumentación conectan los mundos de conocimiento descritos por Tiberghien (2000), estos son el mundo de las teorías y modelos y el mundo de los objetos y eventos. Las autoras concluyen que es necesario prestar más atención a las destrezas de criticar las conclusiones durante el proceso de modelización, ya que la actividad que emplearon, basada en la modelización sobre la expresión de los genes, favorece la elaboración de conclusiones más que la crítica hacia las mismas.

En estas investigaciones los estudiantes trabajaban en grupo, del mismo modo que en el presente estudio. El trabajo en grupo facilita que los estudiantes interactúen en una pequeña comunidad científica en la que se comparte y genera conocimiento científico (Bell Urhahne Schanze y Ploetzner, 2010) como resultado de las interacciones que se producen entre ellos.

\section{Metodología}

Este trabajo sigue una metodología cualitativa por ser un estudio de caso en el cual se analiza el discurso de cuatro grupos de estudiantes $(\mathrm{N}=16)$, que cursaban la asignatura de Biología y Geología de $1^{\circ}$ de bachillerato (16-17 años).

\section{Toma y análisis de los datos}

Los datos fueron obtenidos mediante las grabaciones en audio y video, que posteriormente fueron transcritas, completando esta información con los informes escritos de cada grupo.

Con el fin de analizar la relación entre las prácticas científicas de modelización y argumentación tomamos como base en el marco de análisis presentado por Böttcher y Meisert (2011). El marco de estos autores parte de una representación de la relación entre la modelización y argumentación en una tabla en la cual se recoge: 1) los turnos y los estudiantes, 2) las intervenciones, 3) las aplicaciones de los modelos que hace cada estudiante y 4) los elementos argumentativos que intervienen. No obstante, esta representación ha sido modificada con el fin de ayudar a visualizar mejor los resultados.

El análisis de los datos siguió el siguiente proceso: 1) selección de los episodios de transcripciones en las que se aprecia una revisión de los modelos, 2) identificación de los modelos de los estudiantes y 3) análisis de las destrezas argumentativas que afectan a la construcción, revisión y evaluación de los modelos. Este análisis se presenta en tablas cuya primera columna está destinada a presentar los fragmentos de diálogos. La segunda columna presenta las aplicaciones de los modelos que hace cada estudiante, en la cual las flechas pretenden enfatizar la evolución de los modelos identificados en las explicaciones hasta llegar al adoptado por el grupo. En la tercera columna se resumen las destrezas argumentativas identificadas como sigue: 1) contraargumentar, cuando el sujeto revisa las pruebas desde diferentes puntos de vista, 2) refutar, cuando se rechaza un modelo a partir de las pruebas disponibles, (Kuhn 1991, Justi 2015) 3) justificar, cuando emplean información (datos o pruebas) para apoyar la conclusión y 4) concluir cuando enuncian la explicación final que responde al dilema planteado (Jiménez Aleixandre 2010). 


\section{La propuesta didáctica: ¿Cuál es la verdadera historia del Sinclinal de $\mathrm{O}$ Courel?}

La propuesta didáctica (Anexo I) comprende un problema auténtico en que el alumado debe reunir toda la información disponible para generar una explicación a la formación del pliegue sinclinal de O Courel, una formación singular geológica de Galicia (España), es decir, deben elaborar la historia geológica de dicho pliegue sinclinal. Esta actividad conforma una secuencia didáctica (Blanco Anaya 2015) en la cual los estudiantes abordaban los métodos de datación e historia geológica.

El modelo que los estudiantes tienen que elaborar es de bistoria geológica de un sinclinal. La complejidad de este modelo reside en que deben manejar nociones relacionadas con el tiempo geológico, tanto el tiempo absoluto (deep time), como el relativo (principios estratigráficos) (King 2008). La noción de tiempo geológico es de compleja comprensión, tal y como pusieron de manifiesto varios estudios con estudiantes de diferentes etapas (Ault 1982, Trend 1998). Según Berjillos y Pedrinaci (1994), llegar una comprensión del tiempo geológico requiere integrar ideas de "cambio geológico, facies, sucesión causal y cronología" (p. 242), a lo cual se contribuye con esta actividad puesto que el alumnado tiene que relacionar el ambiente marino de sedimentación (el mar que separaba las dos partes continentales que hoy conforman Galicia) con los fósiles de origen marino que se encuentran en una parte del sinclinal. A su vez, han de organizar la sucesión de los hechos desde ese ambiente marino a un ambiente terrestre y elevado sometido a procesos de erosión, para lo cual los datos tomados de las rocas y fósiles presentes en ese lugar nos sirven de evidencias para interpretar la historia de la Tierra (Loarces, Ferrer y García 2019).

En el caso que nos ocupa, la elaboración de un modelo de historia geológica de un sinclinal necesita de modelos intermedios (Clement, 2008) y de un conjunto de datos, como se detalla a continuación y se resume en la figura 2 .

Modelos intermedios, es decir, modelos de complejidad inferior que conducen a modelo objeto de estudio (Clement, 2008), es decir, de la historia geológica del pliegue sinclinal. Estos modelos intermediarios son: principio de superposición de los estratos y geocronología (M1), tectónica de placas (M2) y erosión (M3). La aplicación que cada estudiante hace de estos modelos (modelos alternativos), se representa en la segunda columna (de la tabla comentada anteriormente) de análisis con la abreviatura del modelo y una letra minúscula según el orden de aparición (ej.: M1a, M1b, M2a, M2b...).

Información sobre el sinclinal (datos). Los datos proporcionados a los estudiantes proceden de 1) la noticia de prensa, 2) las investigaciones geológicas de Matte (1968) para elaborar la tabla de rocas predominantes, indicando la potencia de los estratos, y 3) del estudio de Sanz, Expósito y Montesinos (2000) para elaborar las fichas de los fósiles. También se les adjunta una tabla cronoestratigráfica. 


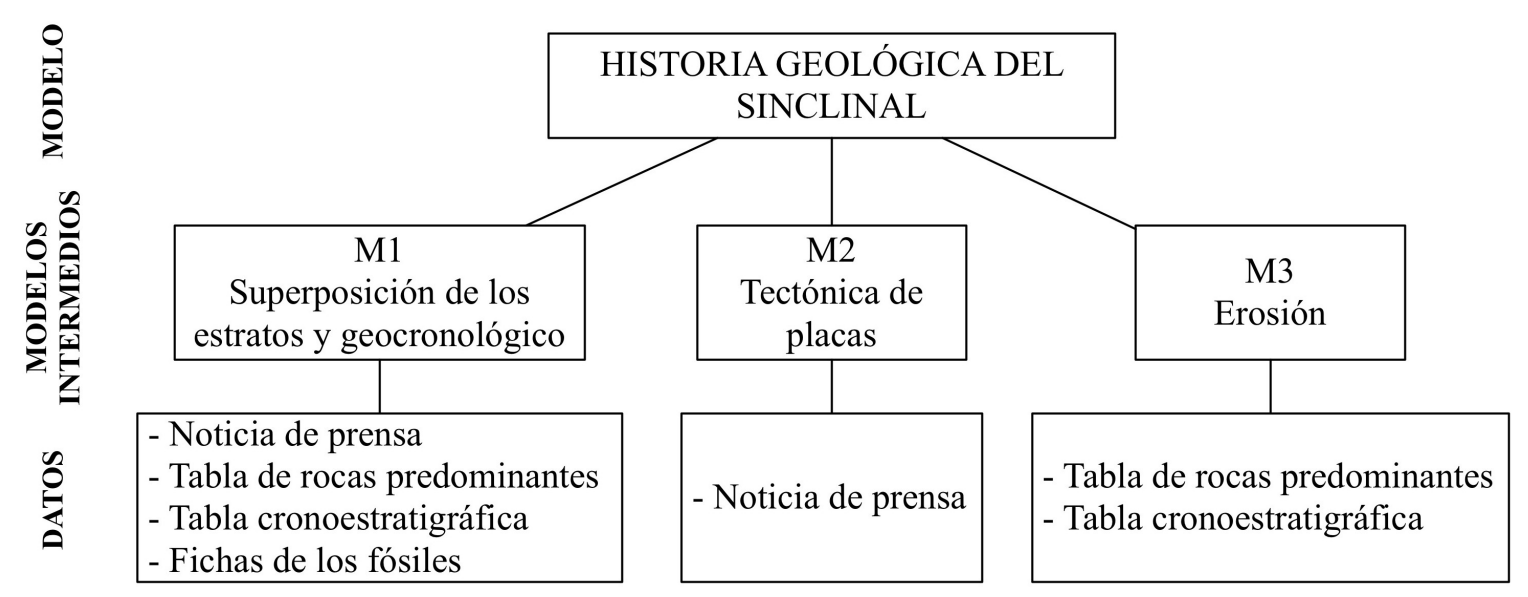

Figura 2. Esquema de la relación entre los modelos intermedios y los datos proporcionados.

Los datos proporcionados, que pueden actuar como prueba, son cruciales para que los estudiantes dispongan de información que les permita justificar sus modelos, contraargumentar y refutar. Es decir, destrezas argumentativas con las que construir, revisar y evaluar su modelo de formación del pliegue sinclinal.

\section{Resultados y discusión}

De los cuatro grupos analizados, sólo dos de ellos han mostrado un discurso argumentativo que constituya una revisión y evaluación de los modelos. En este estudio se detallan los resultados obtenidos del análisis del grupo J, por ser el que mejor retrata esta interacción entre ambas prácticas científicas y porque los datos del grupo $\mathrm{N}$ corroboran los del grupo $\mathrm{J}$.

En el desarrollo de la tarea por el grupo J se han encontrado cuatro momentos en los que las alumnas han modificado sus modelos a través de la argumentación, los cuales se comentan a continuación de forma correlativa durante el desarrollo de la tarea. El primer momento en que discuten (tabla 1) está relacionado con la situación inicial de los continentes, pues en la noticia de prensa les indica que 'Galicia como tal no existía. Estaba separada en dos partes', lo cual Juana y Josefa interpretan de forma diferente. En primer lugar, Juana expresa que los continentes estaban separados y se unieron, haciendo uso del modelo de tectónica de placas al que denominamos M2a (adaptación de M2 'tectónica de placas'). No obstante, Josefa contraargumenta este modelo indicando que el pliegue manifiesta la separación de los mismos (M2b). En ese momento Juana no defiende por qué su modelo es correcto, de modo que las alumnas toman por válido el modelo de Josefa, hasta que ésta se da cuenta de que su modelo es contradictorio con la información indicada en la noticia de prensa, refutando su anterior modelo "Espera porque aquí pone, hace más de 350 millones de años Galicia como tal no existía, estaba separada en dos partes la occidental y la oriental, o sea" (Josefa), y enunciando el M2a expuesto por Juana. Así, el modelo para explicar la situación inicial de los continentes es el M2a, pues como justifica Julia “este plegamiento es la unión de los dos".

El segundo momento que ha supuesto un debate entre las alumnas es cuando establecen la ordenación de los sucesos (tabla 2), es decir, desde la fosilización hasta la aparición de los fósiles en la montaña. Como se puede ver en la primera intervención de ese extracto, Josefa indica que antes del Carbonífero los fósiles están entre los continentes y, tras este periodo, se juntan los continentes. Pero en ese momento Juana propone un contaargumento indicando que "a lo mejor habría que decir cuáles estaban en un trozo y cuales en otro", pues considera que los fósiles ya estaban en los continentes antes de la colisión, lo cual hemos consideramos M1a (adaptación 
del modelo M1 'geocronológico'). Asimismo, de esta intervención de Juana también se puede inferir que está realizando un análisis horizontal de la ordenación de los estratos. Josefa refuta este modelo justificando que los fósiles son marinos, por lo que estaban en el océano (M1b), empleando para ello los datos de las fichas de los fósiles. Debido a que Juana no puede refutar ese argumento de Josefa, pues los datos que tienen indican claramente que los fósiles tienen procedencia marina, Juana adapta esa información a su modelo inicial (M1a), modificándolo a M1c, en el que integra que los fósiles sean marinos porque la montaña estaba cubierta por agua: "En primer lugar, entonces es que la montaña, o lo que fuera estaba cubierta de agua. Y por eso quedaron abí los fósiles pegados”, en sí, esta intervención supone un contraargumento de Juana.

En ese momento Josefa refuta el modelo M1c expuesto por Juana, indicando que "No, porque la montaña no existía que se formó con el choque de las placas, no es que estuviera la montaña abi", justificación en la que aplica el modelo de tectónica de placas (M2). En las siguientes intervenciones las alumnas explican que los fósiles están ordenados en el océano "según la época” (Josefa), pero en cuanto Juana se dispone a redactar la organización de los fósiles en los estratos menciona la posibilidad de que el océano cubriese a la montaña. Josefa vuelve a refutar empleando la misma justificación, pero en esta ocasión es Julia quien consigue que Juana modifique su modelo indicándole "En el fondo del océano habría arena o así, donde quedarían enterrados los diferentes cosos [fósiles]". Así consiguen que Juana cambie su modelo (M1c) por el modelo M1b, relacionándolo con la tectónica de placas (M2), al indicar "Claro, quedaron fosilizados como en el fondo del océano y cuando colisionaron todo vino hacia arriba". La importancia de que Juana modificase su modelo se debe a que es quien está escribiendo y redactando la historia geológica del sinclinal, por ello sus compañeras tratan de que comprenda cómo fue el orden de los sucesos. 
Tabla 1. Evolución del modelo para explicar la situación inicial de los continentes

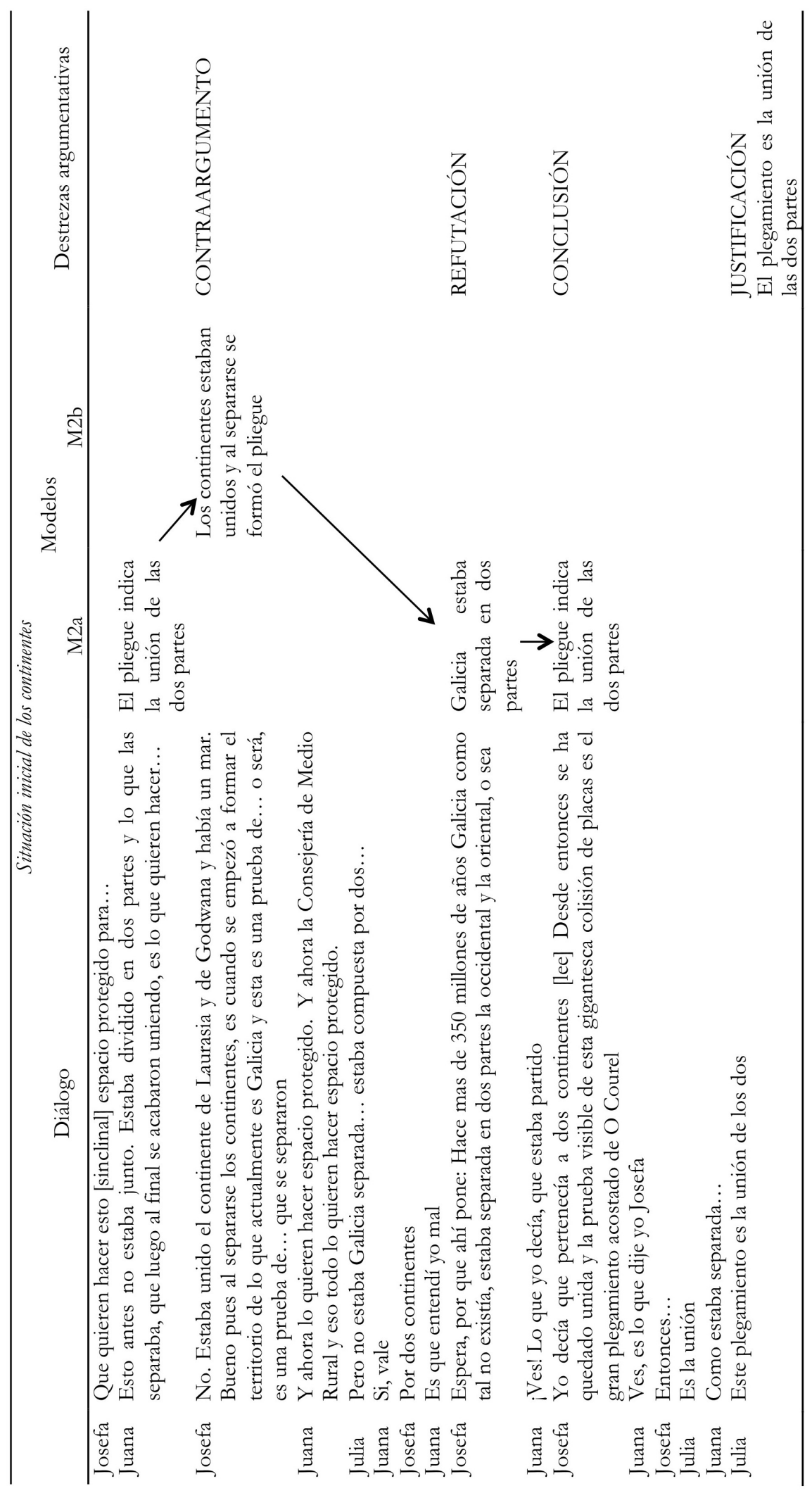


Tabla 2. Evolución del modelo para explicar la ordenación de los sucesos

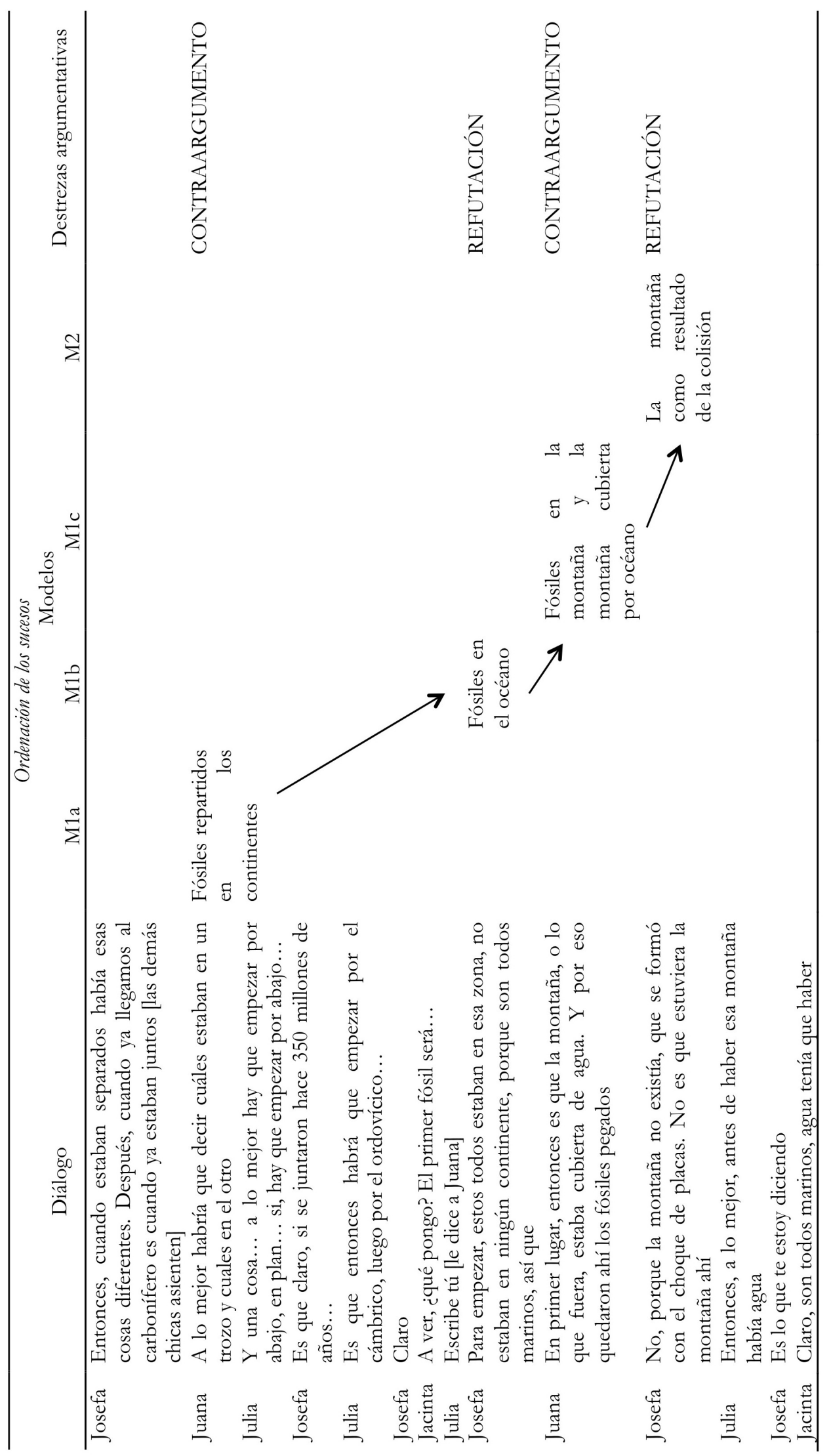


Tabla 2. Continuación

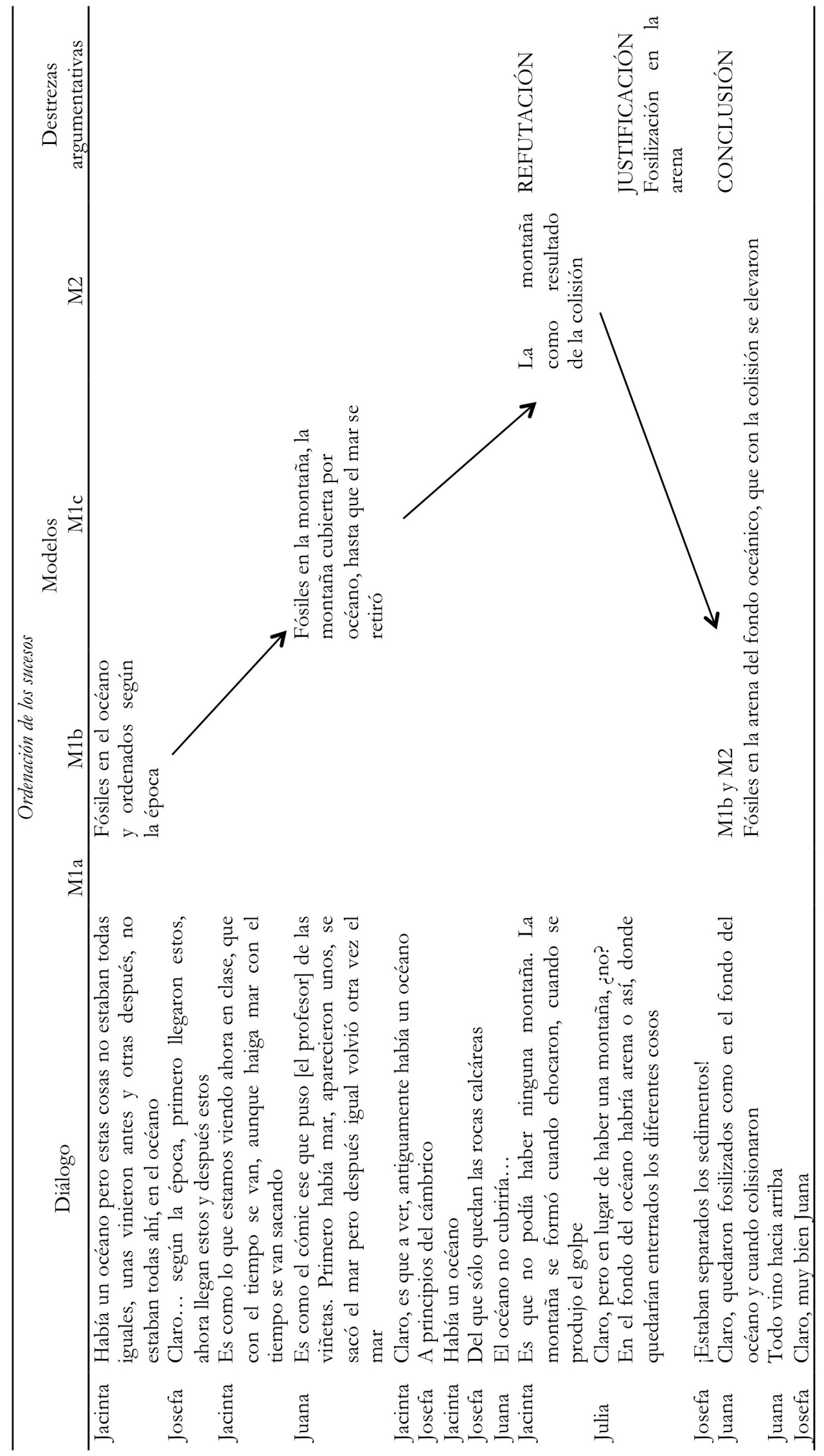


En la tabla 3, la discusión gira entorno a la cronología de los estratos, puesto que cuando Julia y Jacinta proponen que los fósiles más recientes datan del Devónico (M1a), Josefa indica que podría haber más fósiles en el Pérmico, lo cual resulta interesante, pues entiende que pudo haber fosilización después de la formación del pliegue, mostrando una visión dinámica de los procesos geológicos. En cambio, Juana no comprende por qué mencionan el Pérmico, y contraargumenta indicando que el siguiente periodo al Silúrico es el Devónico (M1b), es decir, está interpretando la cronología de los periodos en sentido opuesto, y eso es lo que trata de explicarle Josefa al indicarle que la tabla cronológica se lee hacia arriba y cuando le pregunta: "Juana, ¿qué es antes? hace 570 millones de años o 510" y Julia contribuye a refutar ese contraargumento indicando "Porque antes no se contaba 1000, 1001. Se contaba 1001, 1000", de ese modo Juana indica que comprendió lo que sus compañeras trataban de explicarle, adoptando así el M1a.

El último momento, tabla 4, tratan de acordar la terminología empleada para denominar a la estructura que se forma como resultado del choque de placas, en lo que consideramos que subyace el modelo de tectónica de placas. El M2a es expuesto por Juana al indicar que el choque dio lugar a Galicia. Sin embargo, Jacinta plantea un contraargumento, que consideramos el modelo $\mathrm{M} 2 \mathrm{~b}$, pues indica que el que el choque generó O Courel. Juana agrupa ambas ideas en un nuevo contraargumento en el que indica que tras el choque se formó el Courel y éste dio lugar a Galicia (M2a y b), modelo que es el que queda representado en el informe escrito de la historia geológica, pues es Juana quien está redactando la respuesta. Aunque Josefa y Jacinta no se muestran conformes pues consideran que el resultado del choque fue el pliegue (M2c), refutando los contraargumentos anteriores. No obstante Julia contraargumenta indicando que de no haber choque no habría Galicia y Jacinta contraargumenta de que el choque generó el Courel. El final de la discusión llega cuando Josefa apela a una digresión histórica que está fuera de lugar y cuya única finalidad es persuadir a sus compañeras: "Galicia quedó como Galicia bace pocos años, cuando se murieron los Reyes Católicos, así que no dio lugar a Galicia porque era el reino nosequé, no era Galicia", con ello muestra la dificultad de establecer una relación entre la cronología de los episodios geológicos y la historia reciente del ser humano. 
Tabla 3. Evolución del modelo para explicar la cronología de los estratos

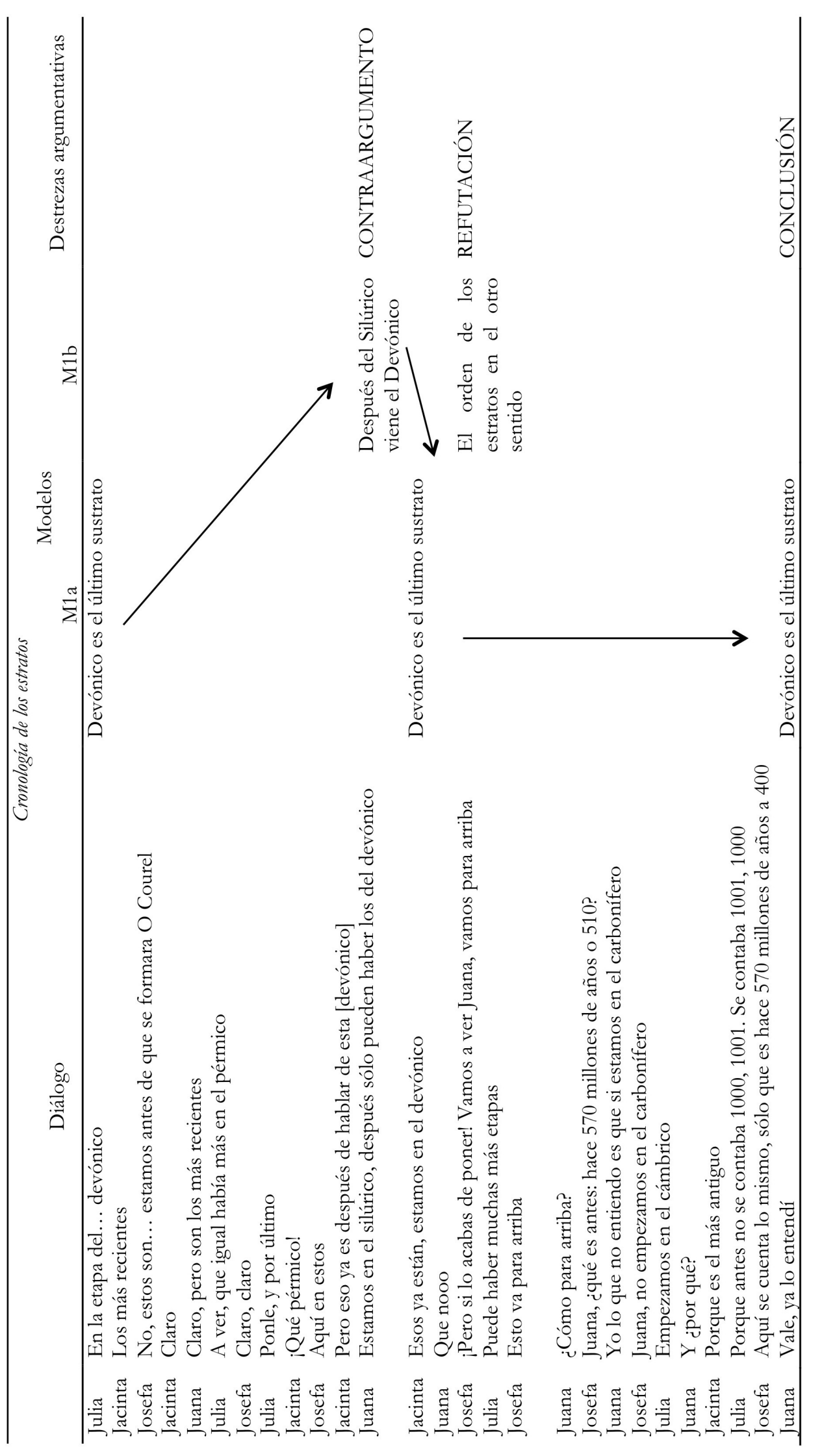


Tabla 4. Evolución del modelo para explicar el resultado del choque

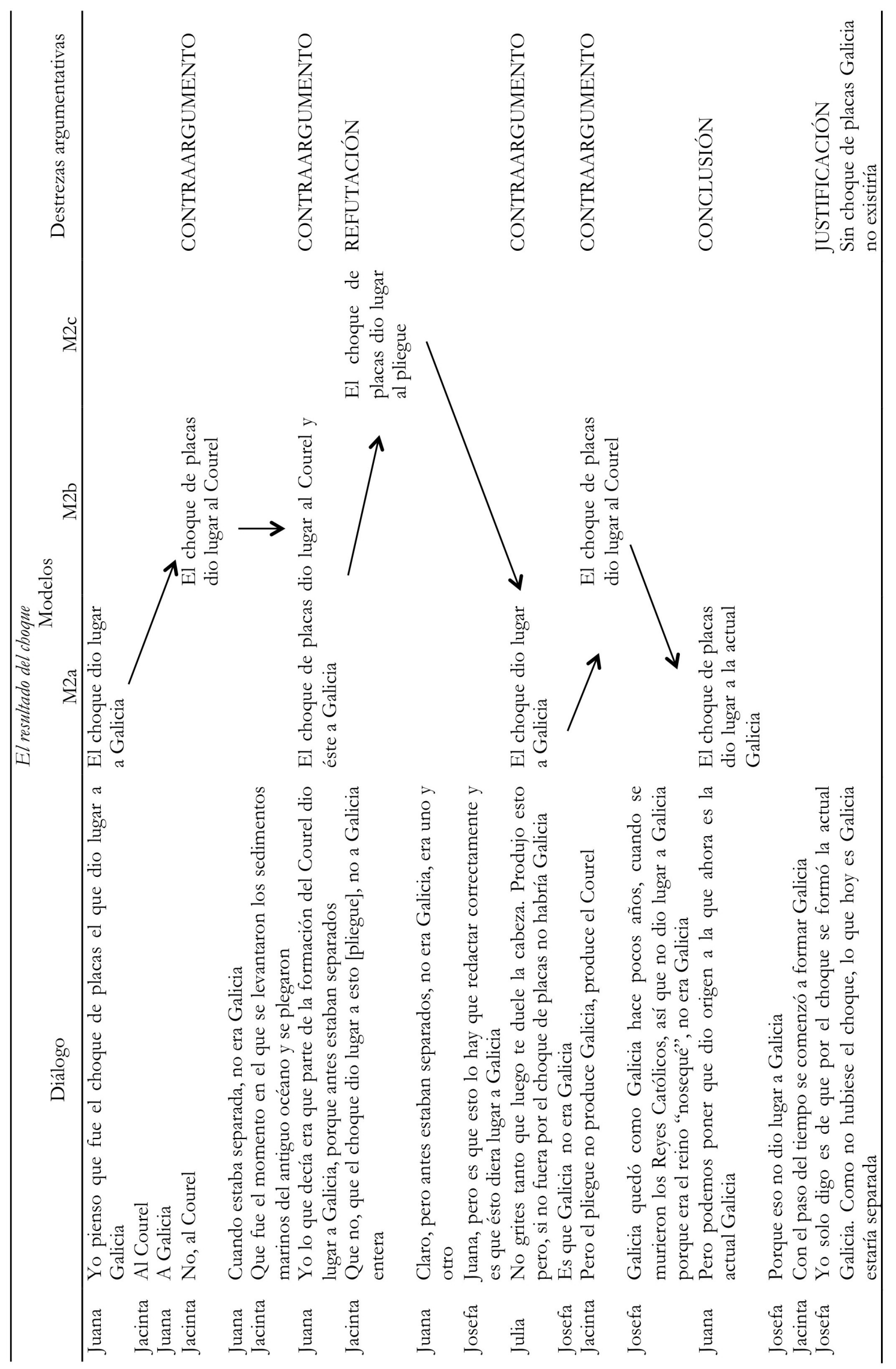




\section{Conclusiones e implicaciones educativas}

El análisis del proceso de modelización seguido por los estudiantes, para dar respuesta a cómo se formó el pliegue sinclinal de O Courel, muestra que este proceso es cíclico, es decir que expresan varios modelos que se evalúan y se vuelven a elaborar mentalmente y expresar verbalmente hasta llegar a los modelos consensuados por el grupo para dar respuesta al fenómeno en cuestión. Considerando el objetivo del estudio, a lo largo de este proceso de modelización se mostraron destrezas argumentativas que contribuyeron a que las alumnas evalúen y cambien sus modelos, en particular los contraargumentos y las refutaciones. Los contraargumentos se han dado cuando las alumnas revisan su modelo inicial pero considerando otros puntos de vista. Asimismo, la destreza de contraargumentar ha sido fundamental cuando los datos presentes no eran suficientes para esclarecer cuál de los modelos que presentaron las alumnas era el más adecuado, por ejemplo ante la discusión a qué dio lugar la colisión de las placas, si a Galicia, al Courel o simplemente al pliegue, para lo cual las alumnas han tenido que emplear sus conocimientos para tratar de persuadir a sus compañeras. En cuanto a las refutaciones han estado presentes en las principales discusiones lo que les ha permitido cambiar los modelos de las participantes. Ello coincide con el estudio de Mendonça y Justi (2014), quienes han encontrado una prevalencia de las destrezas de refutación y contraargumentación en la evaluación de los modelos, pues tal y como "el modelo más apropiado es aquel que logra sobrevivir a las refutaciones gracias a sus justificaciones” (p. 193).

Esto está relacionado con el hecho de que la modelización, así como la argumentación, son fundamentales para desarrollar los conceptos científicos y favorecer el cambio conceptual (Nersessian 2002; Megalakaki y Tiberghien 2011), como en la explicación de la aparición de fósiles en las montañas. Desde la investigación en la didáctica de la geología, este problema conceptual es muy común y ha sido detectado en estudios previos (Pedrinaci 2001). No obstante, conviene considerar si los cambios de modelo de estos estudiantes están restringidos al contexto de esa actividad en particular (Brown, Collins y Duguid 1989) o si es un cambio que dura con el tiempo y es aplicable a otras circunstancias, lo cual se considera que ha sido aprendizaje significativo (Ausubel 2002).

El análisis conjunto de ambas prácticas científicas conlleva una implicación a nivel metodológico, pues las destrezas argumentativas que derivan de los estudios de Kuhn (1991) y de Justi (2015) incluyen la elaboración de teorías alternativas. Si bien en un contexto argumentativo la diferenciación de teorías alternativas y contraargumentos resulta complejo, en el contexto de la modelización consideramos que las teorías alternativas corresponden a los modelos alternativos (M1a, M1b, etc.) que expresan las alumnas. Éstas manifestaron modelos alternativos a los suyos propios, y a los de sus compañeras a medida que analizaban las pruebas disponibles, de forma análoga a las teorías alternativas propuestas por Kuhn (1991). Estas teorías o modelos alternativos han sido evaluados por los contraargumentos y refutaciones ya comentadas.

A diferencia de los grupos cuyo análisis no se incluye en este artículo por ausencia de un proceso argumentativo que favorezca la revisión de los modelos, el grupo que aquí se presenta se caracteriza por 1) hacer explícitos sus modelos, compartiéndolos con las compañeras lo cual ayuda a modificarlos y completarlos, como se ha concluido en otros estudios (Blanco y Díaz 2017); y 2) por un constante intento de persuasión aportando pruebas que refuerzan sus argumentos, contraargumentos y, en consecuencia, sus modelos. En los restantes grupos la ausencia de cambios en los modelos podría venir condicionada por la condescendencia del alumnado con el modelo propuesto por alguno de los integrantes, de tal manera que ese integrante resuelve la actividad. Además, en ninguno de los grupos hubo intervención del docente, lo que nos lleva a considerar que el papel del mismo podría ser importante para 
promover el discurso argumentativo. La ausencia del intento de persuasión y, por lo tanto, la ausencia de manifestación de destrezas argumentativas, coincide con lo encontrado en estudios anteriores, como es el caso de Blanco y Díaz (2014), quienes analizaron el discurso argumentativo de varios grupos concluyendo que la ausencia de episodios de persuasión repercute en la falta de contraargumentos y de refutaciones. Los resultados del estudio de Puig et al. (2017) manifiestan pocas operaciones argumentativas relacionadas con la crítica pues, como señalan las autoras, los estudiantes presentan dificultades para evaluar sus modelos. El intento de persuasión favorece la evolución en el uso de los modelos, ya que va acompañada de contraargumentos $\mathrm{y}$, principalmente, refutaciones fundamentadas en las pruebas disponibles. Trabajos como Mendonça y Justi (2013) y Justi (2015) ponen de manifiesto que la argumentación no sólo permite la evaluación del modelo, como etapa final del proceso de modelización, sino que está presente a lo largo del proceso de justificación del modelo, de revisión y de su uso para generar una explicación. Coincidimos con Puig et al. (2017) en que el diseño de actividades de modelización debe prestar más atención a aspectos que favorezcan el desempeño de las dos prácticas científicas conjuntamente.

Este trabajo manifiesta la necesidad de mejorar el conocimiento acerca del 1) papel del profesor en la regulación de la modelización, con el objetivo de promover, apoyar y mejorar la argumentación, así como del 2) papel de la crítica en la evaluación de los modelos, pues el énfasis en las propuestas didácticas se encuentra más en la construcción de conocimiento que en la evaluación del mismo, lo que implicaría un papel más activo del alumnado.

Desde nuestro punto de vista, la actividad de este estudio fue diseñada para solicitar al alumnado que genere una explicación científica para la formación de un sinclinal, lo que requería la aplicación de varios modelos y, a su vez, se proporcionaron un conjunto de datos necesarios para que el proceso argumentativo fuese fructífero. Por ello, consideramos que diseñar propuestas didácticas basadas en la modelización y argumentación promueve que el alumnado tenga que aplicar su conocimiento científico, mediante la modelización, y ser capaces de justificar sus conclusiones para contribuir a la evaluación de los modelos empleados, generando explicaciones más consistentes de los fenómenos científicos.

\section{Agradecimientos}

$\mathrm{Al}$ alumnado y docentes que participaron en el estudio.

Al proyecto EDU2015-66643-C2-2-P, del Ministerio de Economía y Competitividad de España.

A la Universidade Federal de Ouro Preto, quien concedió la licencia como profesor visitante en la USC a Paula Cristina Cardoso Mendonça.

\section{Referencias}

Ageitos N., Puig B., Calvo Peña X. (2017) Trabajar genética y enfermedades en secundaria integrando la modelización y la argumentación científica. Revista Eureka sobre Enseñanza y Divulgación de las Ciencias 14 (1), 86-97. http://hdl.handle.net/10498/18848

Ault C. R. (1982) Time in geological explanations as perceived by elementary school students. Journal of Geological Education, 30, 304-309. DOI: 10.5408/0022-1368-30.5.304

Ausubel D. P. (2002) Adquisición y retención del conocimiento: una perspectiva cognitiva. Barcelona: Paidós.

Bell T., Urhahne D., Schanze S., y Ploetzner R. (2010) Collaborative inquiry learning: Models, tools, and challenges. International Journal of Science Education, 32(3), 349-377. doi:10.1080/ 09500690802582241 
Berjillos P. y Pedrinaci E. (1994) Concepto de tiempo geológico: orientaciones para su tratamiento en la educación secundaria. Enseñanza de las Ciencias de la Tierra, 2(1), 240251.

Blanco Anaya P. (2015) Modelización y argumentación en actividades prácticas de geología en secundaria (Tesis de doctorado) Universidad de Santiago de Compostela, Santiago de Compostela.

Blanco Anaya P., Díaz de Bustamante J. (2014) Argumentación y uso de pruebas: Realización de inferencias sobre una secuencia de icnitas. Enseñanza de las ciencias, 32 (2),263-280.

Blanco Anaya P. y Díaz de Bustamante, J. (2017) Análisis del nivel de desempeño para la explicación de fenómenos de forma científica en una actividad de modelización. Revista Eureka sobre Enseñanza y Divulgación de las Ciencias 14 (3), 505-520

Böttcher F., Meisert A. (2011) Argumentation in science education: A model- based framework. Science \& Education, 20 (2), 103-140. https://doi.org/10.1007/s11191-0109304-5

Boulter C., Buckley B. (2000) Constructing a typology of models for science education. En: J. K. Gilbert, C. J. Boulter (Eds.), Developing models in science education (pp. 41-57). Dordrecht. Kluwer Academic Publisher.

Brown J. S., Collins A., Duguid P. (1989) Situated cognition and the culture of learning. Educational Researcher, 18, 32-42. https://doi.org/10.3102/0013189X018001032

Clement J. (1989) Learning via Model Construction and Criticism - Protocol evidence on sources of creativity in science. En J.A Glover, R.R. Ronning, C.R. Reynolds, (Eds.), Handbook of Creativity, pp. 341-381. Nueva York: Plenum.

Clement J. J. (2008) Student/Teacher co-construction of visualizable models in large group discussion. En: J. J. Clement, M. A. Rea-Ramirez (Eds.), Model based learning and instruction in science (pp. 11-22). Dordrecht: Springer.

Erduran S., Jiménez-Aleixandre M.P. (2008) Argumentation in science education: perspectives from classroom-based research. Dordrecht, the Netherlands: Springer

Evagorou M., Puig Mauriz B. (2017) Engaging elementary school pre-service teachers in modeling a socioscientific issue as a way to help them appreciate the social aspects of science. International Journal of Education in Mathematics, Science and Technology, 5 (2), 113123. DOI: $10.18404 /$ ijemst.99074

Franco C., Colinvaux D. (2000) Grasping mental models. En J. K. Gilbert, C. J. Boulter (Eds.), Developing Models in Science Education (pp. 93-118). Dordrecht, The Netherlands: Kluwer.

Galagovsky L. R., Adúriz-Bravo A. (2001) Modelos y analogías en la enseñanza de las ciencias naturales. El concepto de modelo didáctico analógico. Enseñanza de las Ciencias, 19 (2), 231-242.

Giere R. N. (2004) How models are used to represent reality. Philosophy of science, 71 (5), 742752.

Gilbert J. K., Boulter C. J., Elmer R. (2000) Positioning models in science education and in design and technology education. En J. K. Gilbert y C. J. Boulter (Eds.), Developing Models in Science Education (pp. 3-17). Dordrecht, The Netherlands: Kluwer. 
Gilbert J.K., Pietrocola M., Zylbersztajn A., Franco C. (2000) Science and Education, Notions of Reality, Theory and Models. En J. K. Gilbert, C. J. Boulter (Eds.), Developing models in science education (pp. 19-40). Dordrecht. Kluwer Academic Publisher.

Gilbert J. K., Justi R. (2016) Modelling-based Teaching in Science Education. Basel, Switzerland: Springer International Publishing.

Jiménez Aleixandre M. P., Bravo B., Puig B. (2009) ¿Cómo aprende el alumnado a usar y evaluar pruebas? Aula de Innovación Educativa, 186, 10- 12.

Jiménez Aleixandre M. P. (2010) 10 Ideas Clave. Competencias en argumentación y uso de pruebas. Barcelona: Graó.

Jiménez-Aleixandre M.P., Crujeiras B. (2017) Epistemic Practices and Scientific Practices in Science Education. En K.S. Taber, B. Akpan (Eds), Science Education. New Directions in Mathematics and Science Education (pp. 69-80). SensePublishers: Rotterdam

Justi R., Gilbert J. K. (2002) Modelling, teachers' views on the nature of modelling, and implications for the education of modellers. International Journal of Science Education, 24 (4), 369-387. https://doi.org/10.1080/09500690110110142

Justi R. (2015) Relações entre argumentação e modelagem no contexto da ciência e do ensino de ciências. Ensaio Pesquisa em Educação em Ciências, 17, 31-48. http://dx.doi.org/10.1590/1983-2117201517s03

King C. (2008). Geoscience education: an overview, Studies in Science Education, 44(2), 187-222. DOI: $10.1080 / 03057260802264289$

Koslowski B., Marasia J., Chelenza M., Dublin R. (2008) Information becomes evidence when an explanation can incorporate it into a causal framework. Cognitive Development, 23 (4), 472-487. https://doi.org/10.1016/j.cogdev.2008.09.007

Kuhn D. (1991) The skills of argument. Cambridge: Cambridge University Press.

Loarces R. G., Ferrer G. F., García F. G. (2019) Evolución de los modelos mentales sobre fosilización tras el proceso de enseñanza-aprendizaje. Revista Eureka sobre Enseñanza y Divulgación de las Ciencias 16(2), 2102 doi: 10.25267/Rev_Eureka_ensen_divulg_cienc.201 9.v16.i2.2102

Márquez, C., Artés, M. (2016) Propuesta de análisis de representaciones sobre el modelo cambio geológico del alumnado del grado de educación primaria. Enseñanza de las Ciencias de la Tierra, 24(2), 169-181.

Matte P. (1968) La structure de la virgation hercynienne de Galice (Espagne). Géologie Alpine, 44, 157-280.

McNeill K. L., Krajcik J. (2008) Inquiry and scientific explanations: Helping students use evidence and reasoning. Science as inquiry in the secondary setting, 121-134.

McNeill K. L., Krajcik J. (2012) Supporting grade 5-8 students in constructing explanations in science: The claim, evidence and reasoning framework for talk and writing. New York, NY: Pearson Allyn \& Bacon.

Megalakaki O., Tiberghien A. (2011) A qualitative approach of modelling activities for the notion of energy. Electronic Journal of Research in Educational Psychology, 9 (1), 157-182. 
Mendonça P. C. C., Justi R. (2013) The relationships between modelling and argumentation from the perspective of the model of modelling diagram. International Journal of Science Education, 35 (14), 2407-2434.

Mendonça P. C. C., Justi R. (2014) An instrument for analyzing arguments produced in modeling-based chemistry lessons. Journal of Research in Science Teaching, 51 (2), 192-218.

Nersessian N. J. (2002) The cognitive basis of model-based reasoning in science. In: P. Carruthers, S. Stich and M. Siegal The Cognitive Basis of Science (pp. 133-153). Cambridge: Cambridge University Press.

Oliva J. M. (2011) Cómo usar analogías en la enseñanza de los modelos y de los procesos de modelización en ciencias. Alambique: Didáctica de las Ciencias Experimentales, 17 (69), 80-91.

Oliveira D. K. B., Justi R., Mendonça P. C. C. (2015) The use of representations and argumentative and explanatory situations. International Journal of Science Education, 37 (9), 1402-1435.

Osborne J. (2011) Science teaching methods: a rationale for practices. School Science Review, 93 (343), 93-103

Osborne J. (2014) Teaching scientific practices: Meeting the challenge of change. Journal of Science Teacher Education, 25 (2), 177-196. https://doi.org/10.1007/s10972-014-9384-1

Passmore C. M., Svoboda J. (2012) Exploring opportunities for argumentation in modelling classrooms. International Journal of Science Education, 34(10), 1535-1554. https://doi.org/10.1080/09500693.2011.577842

Pedrinaci E. (2001) Los procesos geológicos internos. Madrid:Síntesis.

Puig B., Ageitos N., Jiménez-Aleixandre M.P. (2017) Learning Gene Expression Through Modelling and Argumentation, Science \& Education, 26 (10), 1193-1222. https://doi.org/10.1007/s11191-017-9943-x

Reiser B. J., Berland L. K., Kenyon L. (2012) Engaging Students in the Scientific Practices of Explanation and Argumentation. Science and Children, 49 (8), 8-13.

Sanz López J., Expósito Vaqueiro C. M., Montesinos López J. R. (2000) Estratigrafía y conodontos del Devónico Inferior del sinclinal del Caurel-Peñalba (NO de España). En J.B. Díez, A.C. Balbino (Eds.), I Congreso Ibérico de Paleontología, XVI Jornadas de la Sociedad Española de Paleontología y VII International Meeting of IGCP 421, Évora 2000.

Schwarz C. V., Reiser B. J., Davis E. A., Kenyon L., Achér A., Fortus D., Shwartz Y., Hug B., Krajcik J. (2009) Developing a Learning Progression for Scientific Modelling: Making scientific modelling accessible and meaningful for learners. Journal of Research in Science Teaching, 46 (6), 632-654. https://doi.org/10.1002/tea.20311

Siegel H. (1989) The rationality of science, critical thinking and science education. Synthese, 80, 9-41. https://doi.org/10.1007/BF00869946

Stroupe D. (2015) Describing "science practice" in learning settings. Science Education, 99 (6), 1033-1040. https://doi.org/10.1002/sce.21191

Tiberghien A. (2000) Designing teaching situations in secondary school. En R. Millar, J. Leach, y J. Osborne (Eds.), Improving science education. The contribution of research (pp. 27-47). Buckingham: Open University Press.

Toulmin S. (1958, edición de 1964) The uses of argument. Cambridge:University Press. 
Trend R. (1998) An investigation into understanding of geological time among 10- and 11year-old children, International Journal of Science Education, 20(8), 973988. DOI: $10.1080 / 0950069980200805$

Zembal-Saul C., McNeill K. L., Hershberger K. (2013)What's your evidence? Engaging k-5 students in constructing explanations in science. New York, NY: Pearson Allyn \& Bacon. 


\section{Anexo 1. ¿Cuál es la verdadera historia del sinclinal de O Courel?}

En la Sierra de O Courel, en particular en la parte que pertenece al Ayuntamiento de Quiroga, se pueden apreciar unas estructuras geológicas de gran magnitud que datan de varios millones de año.

Desde hace años esta estructura es visitada por numerosos visitantes y Geólogos de varios puntos de Europa. Algunos de estos Geólogos investigaron la geología de la Sierra de O Courel, pero cada uno dentro de su propia disciplina (Litología, Paleontología...).

Ante esa afluencia de visitantes el Ayuntamiento de Quiroga instaló un mirador en la Campodola, pero ahora quiere dar un paso más, dotar a este mirador con un panel informativo en el que se narre la historia geológica más relevante de esta Sierra. Para eso pide colaboración a los estudiantes de Geología de Galicia, pues considera que es una buena forma de dar a conocer esta formación geológica.

El problema es que disponemos de varias piezas de información que se pueden ver a continuación. Por lo tanto, debéis reunir esas piezas y reconstruir la historia geológica de la sierra, de una forma simple e indicando los materiales que la forman.

Información de la que disponéis:

- Noticia de prensa

- Tabla de las eras y rocas predominantes

- Fichas de los fósiles encontrados

- Tabla cronoestratigráfica

Pregunta final: ¿por qué no hay más datos registrados a partir del Devónico? 